Journal of Computer Science 6 (10): 1154-1158, 2010

ISSN 1549-3636

(C) 2010 Science Publications

\title{
Speckle Suppression of Radar Images Using Normalized Convolution
}

\author{
${ }^{1}$ A.K. Helmy and ${ }^{2}$ G.S. El-Taweel \\ ${ }^{1}$ National Authority of Remote Sensing and Space Science, Cairo, Egypt \\ ${ }^{2}$ Department of Computer Science, Faculty of Computers and Informatics, \\ Suez Canal University, Ismailia, Egypt
}

\begin{abstract}
Problem statement: Synthetic Aperture Radar (SAR) images are becoming more widely used in remote sensing applications. SAR uses microwave radiation to illuminate the earth's surface. The coherent microwave illumination, however, suffers from fading effects result in generating a multiplicative speckle noise that corrupts SAR images. Approach: Through this study, scheme to reduce speckles was proposed, it was designed to accomplish the following goals: Reduction of variance in homogeneous areas, side by side with preservation of edges and lines. Our scheme estimates data samples where speckles were resided from samples on regularly shifted sampled grid of original SAR image. Firstly, median filter was first applied on the original SAR image. Secondly, edge enhanced filter was applied to the median filtered image. Then the filtered images (median and edge enhanced median images) were spatially shifted a with respect to the original SAR image. Lastly, Noise-free SAR image was derived through a projection of down sampled shifted images (median filtered, edge enhanced of median filtered and original SAR Image) onto a subspace using Normalized Convolution (NC). This led to more samples of the same modality being gathered for the analysis (smoothness through applying median filter, edges from enhancement of median filter and original SAR samples), which in turn improves signal-to-noise ratio and reduces diffusion across discontinuities. Results: Proposed scheme produces same variance level in smoothed area as other statistical filter, about 0.12 higher than the best of statistical filter. On the other hand it surplus other statistical filters in preserving fine detail as it produce 33 and 27\% higher than the best reading of the statistical filter in ENL and DR values respectively. Conclusion: The proposed scheme produced compromise results of speckles reduction and preserving level of fine details that have an important factor in further image processing and interpretation.
\end{abstract}

Key words: SAR image, speckle reduction, normalized convolution

\section{INTRODUCTION}

Speckles affect all coherent imaging systems including laser, medical ultrasound and SAR imagery. Recently, there has been considerably interest in using the wavelet transform as a powerful tool for recovering SAR images from noisy data (Guo et al., 1994; Gagnon and Jouan, 1997). Basically, all wavelet based methods involve as a first step the use of a logarithmic transform to separate the noise from the original image. Then, different wavelet shrinkage approaches are adopted, which are based on Donoho (1995) pioneering work. SAR images filtering also require a good preservation of textural features. It has shown that a successful imaging algorithm can achieve both noise reduction and feature preservation if it takes into consideration the true statistics of the signal and noise components (Melnik et al., 2000; Aglika, 2002).
Speckle is caused by the interference between waves reflected from microscopic scattering through the terrain, in the particular case of SAR images. This kind of noise can be modeled in terms of a random walk in the complex plane and, in the case of a large number of scatters within the resolution cell (fully developed speckle); the real and imaginary parts of the resulting complex field are Gaussian random variables. It has been experimentally verified in several works that over homogeneous areas, the standard deviation of the signal is proportional to its mean (Gagnon and Jouan, 1997).

Methods used previously to reduce speckle noise in images include the local statistics method (Melnik et al., 2000), the sigma filter (Lee, 1981), the median filter (Fisher et al., 2001), homomorphic filtering (Mark, 1995) and adaptive linear smoothing (Achim et al., 2001). Although most of these

Corresponding Author: A.K. Helmy, National Authority of Remote Sensing and Space Science, Cairo, Egypt 
techniques exhibit at least some degree of edge preservation, the results of these filters generally either still have too much noise or do not have distinct enough edges to allow simple edge detection algorithms to perform well (Gagnon and Jouan, 1997).

Statistical properties of radar speckle noise: The statistical properties of speckle noise were studied by Goodman (1996). He has shown that, if the number of scatterers per resolution cell is large, a fully developed speckle pattern can be modeled as the magnitude of a complex Gaussian field with Independent and Identically Distributed (IID) real and imaginary components. Arsenault and April (2000) have shown that when the image intensity is logarithmically transformed, the speckle noise is approximately Gaussian additive noise and it tends to a normal probability much faster than the intensity distribution. Consequently, we use the log-normal distribution as a speckle noise model: if $\mathrm{x}$ follows the log-normal distribution with certain mean and variance values, then $\ln \mathrm{x}$ follows the normal distribution with the same mean and the same variance. A lognormal random variable can be generated using:

$$
X_{\text {log-normal }}=\exp \left(X_{\text {normal }} \sqrt{\left.2 \log \frac{M}{m}+\ln m\right)}\right.
$$

Where:

$\mathrm{M}$ and $\mathrm{m}=$ The mean and the median values of the distribution, respectively

$\mathrm{X}_{\text {normal }}=\mathrm{A}$ standard zero mean, unit-variance Gaussian random variable

There is a straightforward equivalence between the Equivalent Number of Looks (ENL) in a speckle image and the parameters in Eq. 1.

Normalized convolution: Normalized Convolution (NC) (Knutsson and Westin, 1993; Kuan et al., 1987) is a technique for local signal modeling from projections onto a set of basis functions. Although any bases can be /"[lmjm, used, the most common one is a polynomial basis: $\left\{1, \mathrm{x}, \mathrm{y}, \mathrm{x}^{2}, \mathrm{y}^{2}, \mathrm{xy} \ldots\right\}$, where $1=\left[\begin{array}{ll}1 & 1 \ldots 1\end{array}\right]^{\mathrm{T}}(\mathrm{N}$ entries), $x=\left[\begin{array}{ll}x_{1} x_{2} \ldots x_{N}\end{array}\right]^{\mathrm{T}}, x^{2}=\left[x^{2}{ }_{1} x_{2}^{2} \ldots . x_{N}^{2}\right]^{T}$ and so on are constructed from local coordinates of $N$ input samples. The use of polynomial basis functions makes the traditional $\mathrm{NC}$ equivalent to a local Taylor series expansion. Within a local neighborhood centered at $\mathrm{s}_{0}$ $=\left\{\mathrm{x}_{0}, \mathrm{y}_{0}\right\}$, the intensity value at position $\mathrm{s}=\left\{\mathrm{x}+\mathrm{x}_{0}, \mathrm{y}\right.$ $\left.+\mathrm{y}_{0}\right\}$ is approximated by a polynomial expansion:

$$
\begin{aligned}
\hat{\mathrm{f}}\left(\mathrm{s}, \mathrm{s}_{0}\right) & =\mathrm{p}_{0}\left(\mathrm{~s}_{0}\right)+\mathrm{p}_{1}\left(\mathrm{~s}_{0}\right) \mathrm{x}+\mathrm{p}_{2}\left(\mathrm{~s}_{0}\right) \mathrm{y} \\
& +\mathrm{p}_{3}\left(\mathrm{~s}_{0}\right) \mathrm{x}^{2}+\mathrm{p}_{4}\left(\mathrm{~s}_{0}\right) \mathrm{xy}+\mathrm{p}_{5}\left(\mathrm{~s}_{0}\right) \mathrm{y}^{2}
\end{aligned}
$$

Where:

$\{x, y\}=$ The local coordinates of sample s with respect to the center of analysis $\mathrm{s}_{0}$

$\mathrm{p}\left(\mathrm{s}_{0}\right)=\left[\mathrm{p}_{0} \mathrm{p}_{1} \ldots \mathrm{p}_{\mathrm{m}}\right]^{\mathrm{T}}$

$\left(\mathrm{s}_{0}\right) \quad=$ The projection coefficients onto the corresponding polynomial basis functions at $\mathrm{s}_{0}$

Different from the Haralick and Watson (1981) facet model, which is also a polynomial expansion, NC uses a so-called applicability function to localize the polynomial fit (while the facet model gives an equal weight to all samples in a neighborhood). This applicability function is often an isotropic, radially decaying function whose size is proportioned to the scale of analysis. A Gaussian function is often used for this purpose. The projection $\mathrm{p}\left(\mathrm{s}_{0}\right)$ can then be used to derive Gaussian derivatives, which are image projections onto Hermite polynomials (Young, 2001). In addition, $\mathrm{NC}$ allows each input signal to have its own certainty value. The signal certainty is especially useful when data samples are missing or are unreliable (e.g., due to bad sensors or erroneous registration). Both the applicability function and the signal certainty control the impact of a particular sample to the local polynomial fit. The choice of the polynomial order depends on specific applications. If processing speed is more important than accuracy, $\mathrm{NC}$ with a constant basis is sufficient. This locally flat model, however, does not model edges and ridges very well. First-order NC with three bases $\{1, \mathrm{x}, \mathrm{y}\}$ can model edges and second-order $\mathrm{NC}$ with six bases $\left\{1, \mathrm{x}, \mathrm{y}, \mathrm{x}^{2}, \mathrm{xy}, \mathrm{y}^{2}\right\}$ can further model ridges and blobs. Higher-order NC can fit more complex structures at a higher computational cost (Tuan et al., 2010).

In this study, we propose to use second-order NC for noise removal from SAR Images. The scale of the applicability function also plays a decisive role in the quality of interpolation. Low-order NC with a large applicability window cannot reconstruct small details in the image. The scale of the applicability function, however, must be large enough to cover sufficient samples for a stable local analysis. Unless the sample density is high everywhere in the image, a normal choice of the applicability function is a Gaussian function with a spatial scale $\sigma_{\mathrm{s}}=1 \mathrm{HR}$ pixel and a truncation of three standard deviations. This Gaussian applicability function introduces minimal blurring to the interpolation result while its support is still large to cover enough samples.

\section{MATERIALS AND METHODS}

The proposed scheme: In this study, we follow a fourstage approach to remove speckles from SAR image as depicted in Fig. 1. 


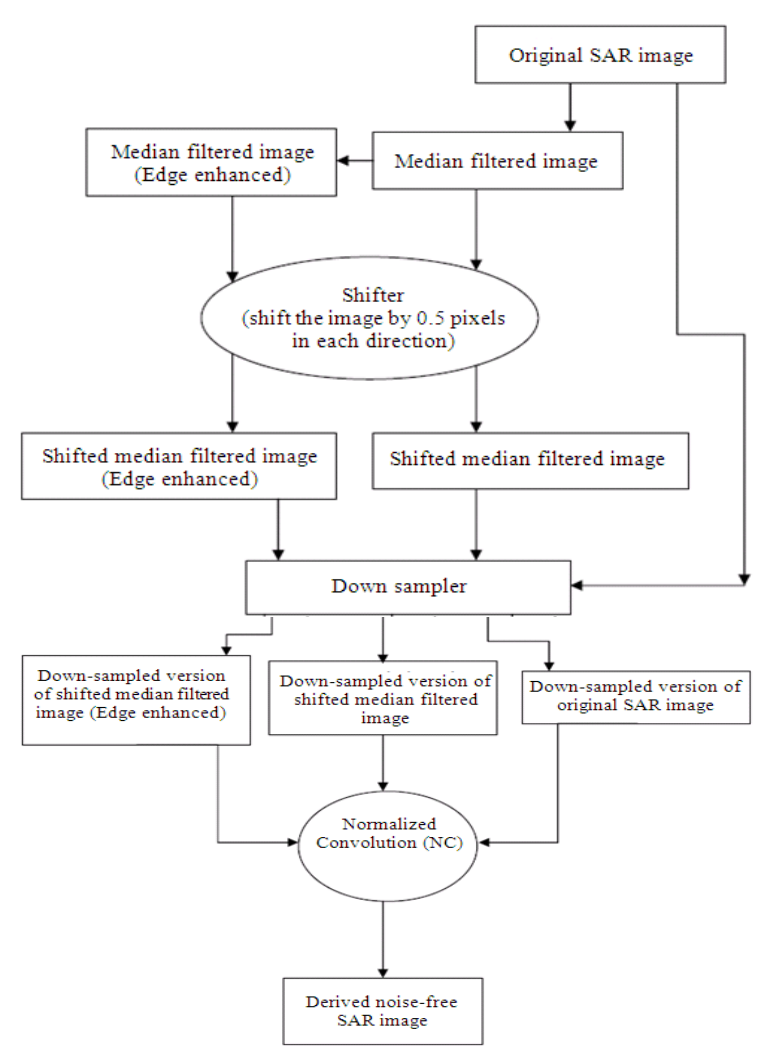

Fig. 1: Flow chart for proposed SAR image de-noise system

Firstly, the original SAR image is subjected to median filter (Fisher et al., 2001) to remove the speckles across the image. Then the result of median filter is subject to edge enhancement filter (Russ, 2002) to modify the over-smooth effects caused by the median filter.

Secondly, the results of filters in previous stage (median filter and edge enhancement filter) are spatially shifted with respect to the original SAR image by value 0.5 pixel in both $\mathrm{x}$ and $\mathrm{y}$ direction.

Thirdly, the original SAR image and the output of second stage are down-sampled by factor 2 in both $\mathrm{x}$ and y direction. Accordingly, we guarantee that we did not loose any information due to down-sampled process (the losses of information in one image is compensated from the original SAR image) Finally, Normalize Convolution is used to merge the two version of SAR images (the output of median filter and edge enhancement filter) added with them the original SAR image to produce noise free one. Thus we make use of all information associated with all inputs.

Performance evaluation of the proposed scheme: In this study, the assessment parameters used to evaluate the performance of speckle reduction are noise variance, equivalent number of looks and deflection ratio (Nason and Silverman, 1995).

Noise variance: Noise variance determines the contents of the speckles in the image. A lower variance gives a 'cleaner' image as more speckles are reduced. The formula for calculating the variance is given as:

$$
\delta^{2}=\left(\frac{1}{\mathrm{~N}} \sum_{1}^{\mathrm{N}-1}\left(\mathrm{X}_{\mathrm{J}}\right)^{2}\right)
$$

Equivalent Numbers of Looks (ENL): Another good approach of estimating the speckle noise level in a SAR image is to measure the Equivalent Numbers of Looks (ENL) over a uniform image region. A larger the value of ENL usually corresponds to a better quantitative performance. The value of ENL also depends on the size of the tested region, theoretically a larger region will produces a higher ENL value than over a smaller region but it also tradeoff the accuracy of the readings. Due to the difficulty in identifying uniform areas in the image, dividing the image into smaller areas of $32 \times 32$ pixels is proposed, obtain the ENL for each of these smaller areas and finally take the average of these ENL values. The formula for the ENL calculation is given in as:

$\mathrm{ENL}=(\mu / \sigma)^{2}$

Where:

$\mu=$ The mean of the uniform region

$\sigma=$ The standard deviation of a uniform region the significance of obtaining both MSE and ENL measurements in this study is to analyze the performance of the filter on the overall region as well as in smaller uniform regions

Deflection Ratio (DR): The last performance measure that we used is in this study is the deflection ratio proposed by (Nason and Silverman, 1995). The formula for the deflection calculation is given as:

$$
\mathrm{M}=\left(\mathrm{X}_{\mathrm{i}, \mathrm{j}}-\overline{\mathrm{X}}_{\mu}\right) / \mathrm{X}_{\sigma}
$$

Where:

$X_{i, j}=$ The scalar pixel value of the image

$\overline{\mathrm{X}}_{\mu}=$ The estimated mean of $\mathrm{X}_{\mathrm{i}, \mathrm{j}}$

$\mathrm{X}_{\sigma}=$ The estimated standard deviation of $\mathrm{X}_{\mathrm{i}, \mathrm{j}}$

The ratio $\mathrm{M}$ should be higher at pixels with stronger reflector points and lower elsewhere. Aglika (2002) use this ratio to measure the performance between different wavelet shrinkage techniques on the diagonal sub-band only. Instead the image is divided 
into smaller areas of $32 \times 32$ pixels, obtain the DR for each of these smaller areas and finally take the average of these DR's values.

\section{RESULTS}

In the results, an experiment is carried out on real SAR data to demonstrate the robust noise removal capability of NC applied to a sequence of input data. The input consists of three $512 \times 512$ images include original SAR image, median filtered and edge enhanced of median filtered image. The last two images are spatially shifted with respect to the original image by 0.5 pixel in each direction. (a)

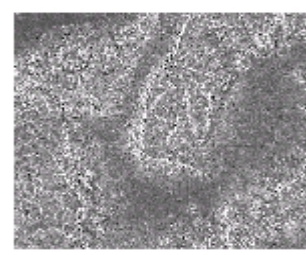

(c)

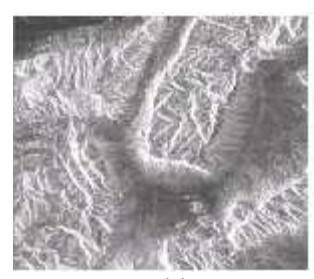

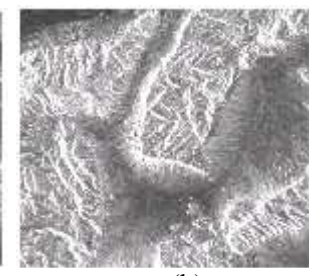

(b)

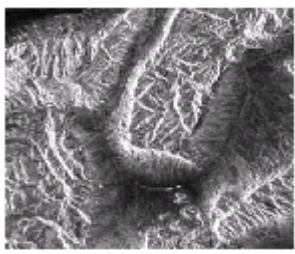

(d)
Fig. 2: Output images at different stages of proposed algorithm; (a) Original SAR image; (b) Median filtered image; (c) High pass filter of median image; (d) Output image

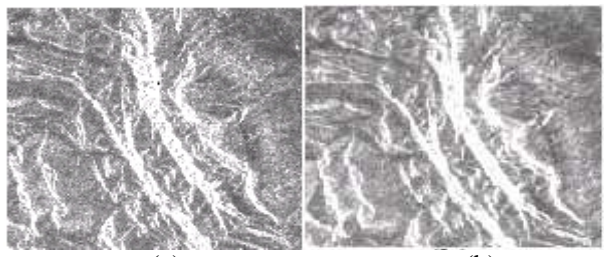

(a)

(b)

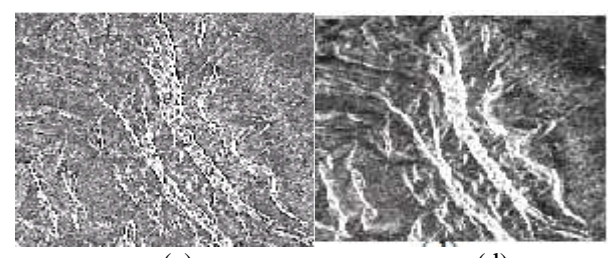

(c)

(d)

Fig. 3: Closer look (from Fig. 2) of output images at different of proposed algorithm with same orders in Fig. 2
For numerical evaluation; variance, ENL and DR have been used side by side with visual inspection. The results of the proposed scheme are compared with sigma-lee, frost and gamma filters (Gagnon and Jouan, 1997; Lee, 1981).

Figure 2 a shows that the speckle noise is prominent in original radar SAR image in both flat and hilly portions of the image; Fig. 3a shows the closer look of the input image. The performance measures used to evaluate the results are listed in Table 1. The de-noised images using proposed scheme and stander statistical filters are shown in Fig. 4.

\section{DISCUSSION}

Table 1 shows that the output of median filter has the lowest values of variance in smoothed area (about $23 \%$ lower than original SAR noise) that implies higher ability to suppress the noise on the other hand, it has an exaggerate values in terms of ENL and DR which result in over-smoothing effects at image details, this is quite clear in Fig. $2 b$ and $3 b$.

The reading analysis of the results and the output denoised images shown in Fig. 2-4 indicate that: All statistical filters superior than the propose scheme in terms of variance, which indicate better ability to reduce the speckles. Mean while the proposed scheme is better than others in terms of ENL and DR indicating better ability of preserving image details. This due to the strong contribution of original image in the output filtered image.

Table 1: Statistical measures for different de-noised filters

\begin{tabular}{llrl}
\hline Measures filters & $\delta^{2}$ & ENL & DR \\
\hline Original SAR image & 8.047 & 7.34 & 1.40 \\
Frost & 6.448 & 11.20 & 2.30 \\
Gamma & 6.338 & 9.40 & 1.34 \\
Median & 6.224 & 6.20 & 1.24 \\
Lee-sigma & 6.408 & 12.50 & 3.45 \\
Proposed & 6.986 & 16.70 & 4.40 \\
\hline
\end{tabular}

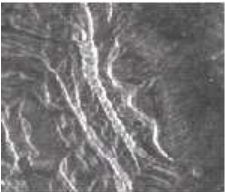

(a)

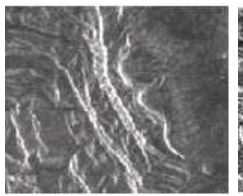

(c)

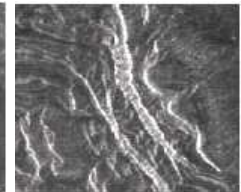

(b)

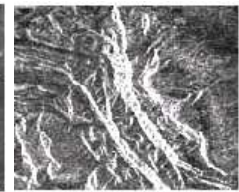

(d)
Fig. 4: De-noised images using proposed algorithm and stander statistical filters. (a) The output using sigma-lee filter (b) The out put of frost filter (c) the gamma filter (d) the output of proposed de-noise filter 


\section{CONCLUSION}

A scheme for de-noise SAR image by applying normalized convolution to the series of pre-processed SAR images in addition to original image is proposed and evaluated in this study.

Compared to other statistical filters, sigma-Lee filter introduces an acceptable compromise between speckle reduction and edge preservation. Comparing the proposed scheme with the best results of statistical filters (Lee-sigma) shows that, it surplus sigma-Lee filters in preserving fine details (ENL and DR), while sigma-lee has a slight improvement in variance values the proposed scheme has a promising results in reducing fading and speckles in radar SAR images since it is quite successful in constructing expansion systems (many other filters can be added the input image sequences) so as to give height sparsity and super resolution but at the coast of added computation and non linearity.

\section{REFERENCES}

Achim, A., A. Bezerianos and P. Tsakalides, 2001. Novel Bayesian multiscale method for speckle removal in medical ultrasound images. IEEE Trans. Med. Image, 20: 772 783, http://portal.acm.org/citation.cfm?id=1279009.127 9195

Aglika, G., 2002. Undecimated wavelet transforms for image denoising. University of Nevada. http://citeseerx.ist.psu.edu/viewdoc/download?doi= 10.1.1.8.1435\&rep=rep1\&type $=$ pdf

Arsenault, H.H. and G. April, 2000. Properties of speckle integrated with a finite aperture and logarithmically transformed. J. Opt. Soc. Am., 66: 1160-1163.

http://portal.acm.org/citation.cfm?id=1057851.105 7888

Donoho, D.L., 1995. Denoising by soft-thresholding. IEEE Trans. Inform. Theory, 41: 613-627. DOI: 10.1109/18.119733

Fisher, R., S. Perkins, A. Walker and E. Wolfart, 2001. Median filter. INF Press. http://homepages.inf.ed.ac.uk/rbf/HIPR2/median.ht $\mathrm{m}$

Gagnon, L. and A. Jouan, 1997. Speckle Filtering of SAR images-a comparative study between complex wavelet based and standard filters. SPIE Proc., 3169: 80-91. DOI: 10.1.1.54.3239
Goodman, J.W., 1996. Some fundamental properties of speckle. J. Opt. Soc. Am., 66: 1145-1150. http://portal.acm.org/citation.cfm?id=1780209

Guo, H., J.E. Odegard, M. Lang, R.A. Gopinath and I.W. Selesnick, 1994. Wavelet based speckle reduction with application to SAR based ATD/R. Proc. Int. Conf. Image Process., 1: 75-79.

Haralick, R.M. and L. Watson, 1981. A facet model for image data. Comput. Graph. Image Proc., 15: 113-129. DOI: $10.1109 / 34.19033$

Knutsson, H. and C.F. Westin, 1993. Normalized and differential convolution. Proceeding of IEEE Computer Society Conference on Computer Vision and Pattern Recognition, (CVPR'93), IEEE Press, New York, USA., pp: 515-523.

Kuan, D.T., A.A. Sawchuk, T.C. Strand and P. Chavel, 1987. Adaptive restoration of images with speckle. IEEE Trans. ASSP, 35: 373-383.

Lee, J.S., 1981. Speckle analysis and smoothing of synthetic aperture radar images. Comput. Graph. Image Process., 17: 24-32. DOI: 10.1016/S0146664X(81)80005-6

Mark, A.S., 1995. Noise reduction in syntactic aperture radar imagery using a morphology-based nonlinear filter. Proceeding of the Digital Image Computing: Techniques and Applications, Conference of Australian Pattern Recognition Society, (APRS'95), IEEE Press, Brisbane, pp: 661-666.

Melnik, V.R., V. Lukin, K. Egiazarian and J. Astola, 2000. A method of speckle removal in one-look SAR images based on lee filtering and wavelet denoising. Proceeding of the IEEE Nordic Signal Processing Symposium, (NORSIG'00), Kolmarden, Sweden, pp: 1-4.

Nason, G.P. and B.W. Silverman, 1995. The Stationary Wavelet Transform and Some Statistical Application. In: Wavelets and Statistics, Antoniadis, A. and G. Oppenhim (Eds.), SpringerVerlag, New York, pp: 281-299.

Russ, J.C., 2002. The Image Processing Handbook. 4th Edn., CRC Press, New York, ISBN: 13: 9780849311420, pp: 732.

Tuan, Q.P., L.J. Van Vliet and K. Schutte, 2010. Robust fusion of irregularly sampled data using adaptive normalized convolution. EURASIP J. Applied Sig. Proc., 2006: 236-236.

Young, R.A., 2001. The Gaussian derivative model for spatial vision: I. Retinal mechanisms. Spat. Vis., 14: 273-293. 